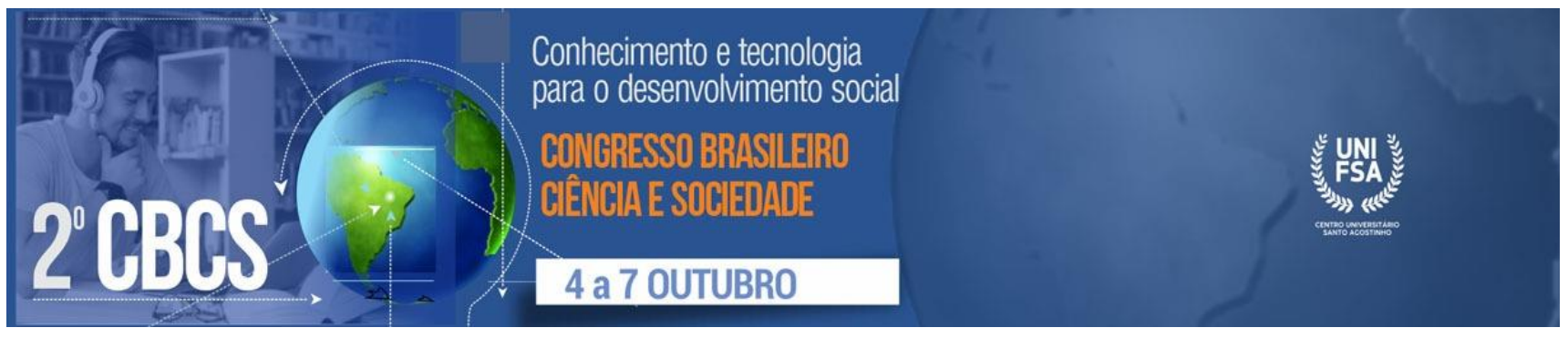

ANAIS CBCS 2021 | 4 a 7 de outubro de 2021 | Centro Universitário Santo Agostinho - Teresina - PI | www.unifsa.com.br/cbcs2021/

\title{
ANSIEDADE EM ESTUDANTES UNIVERSITÁRIOS NO ENSINO REMOTO DURANTE A PANDEMIA DA COVID-19
}

\author{
Patrícia Melo do Monte (UNIFSA) ${ }^{1}$ \\ Walter Emmanoel Brito Neto (UNIFSA) ${ }^{2}$ \\ Francisco Augusto de Brito Vieira (UNIFSA) ${ }^{3}$
}

\begin{abstract}
RESUMO
Objetivo: Abordar acerca das experienciações ansiogênicas de acadêmicos do Ensino Superior, vivenciadas com a instauração do Ensino Remoto Emergencial, com a pandemia da covid-19 no Brasil, expressas na rede social do Twitter. Metodologia: Realizada a partir de um estudo exploratório, qualitativo e documental, na qual se utilizou como material de análise tweets do período de março de 2019 a março de 2020. Resultados e Discussões: As implicações da covid19 ultrapassaram a esfera biológica, enviesando-se sobre os aspectos psicossociais do indivíduo, com o estabelecimento do isolamento social e o fechamentos das instituições de ensino, o Ensino Remoto Emergencial tornou-se uma realidade, implicando, portanto, sobre as vivências universitária de seus usuários, o que ocasionou altos índices de ansiedade e sofrimento psíquico, que devido ao distanciamento, obtiveram expressão por meio das redes sociais, incluindo o Twitter. Conclusão: Observou-se, portanto, uma necessidade de atenção das instituições de saúde e educacionais, quanto a necessidade de amparo e observância dos alunos.
\end{abstract}

Palavras-chave: Ansiedade. Ensino Remoto. EAD. Universitários. Twitter. Pandemia.

\begin{abstract}
Objective: To address the anxiogenic experiences of higher education academics, experienced with the establishment of Remote Emergency Education, with the covid-19 pandemic in Brazil, expressed on the Twitter social network. Methodology: Carried out from an exploratory, qualitative and documentary study, in which tweets from the period from March 2019 to March 2020 were used as material for analysis. Results and Discussions: The implications of covid-19 went beyond the biological sphere, biasing about the psychosocial aspects of the individual, with the establishment of social isolation and the closure of educational institutions, Emergency Remote Teaching became a reality, thus implying on the university experiences of its users, which caused high rates of anxiety and psychological distress, which, due to the distance, were expressed through social networks, including Twitter. Conclusion: It was observed, therefore, a need for attention from health and educational institutions, as the need for support and observance of students.
\end{abstract}

Keywords: Anxiety. Remote Teaching. EAD. College students. Twitter. Pandemic. 


\section{INTRODUÇÃO}

Descrita como um sentimento vago e desagradável de medo, apreensão, caracterizado por tensão ou desconforto derivado de antecipação de perigo, de algo desconhecido ou estranho, a ansiedade, quando se apresenta em excesso, pode ser prejudicial ao sujeito. Ao assumir "sintomas psíquicos negativos contínuos, como irritabilidade, humor depressivo, angústia, baixo limiar para frustrações, fobias, preocupações crônicas, apreensão contínua, obsessão, velocidade exacerbada dos pensamentos", de maneira intensa e duradoura, atrapalha a rotina e atividades cotidianas do sujeito (CASTILLO, RECONDO, ASBAHR E MANFRO, 2000).

É considerada uma resposta a estressores do ambiente e apresenta alta prevalência no contexto atual pandêmico, que exige novas regras e hábitos sociais para a população mundial e mobilizações de diversas naturezas para contenção da doença Covid-19. No que se refere às principais mudanças, destacam-se o distanciamento, o isolamento social e as próprias medidas de biossegurança, pois, levando em consideração o seu caráter indispensável, permearam de forma integral esta nova rotina, logo o automonitoramento frequente associou-se a uma elevação de ansiedade (ROLIM, 2020).

Em consequência da Covid-19 e seus reais riscos de saúde pública, os sistemas educacionais foram afetados, acarretando o fechamento generalizado de instituições de ensino como escolas, faculdades e universidades (UNESCO, 2020). Medidas foram tomadas a fim de se criar alternativas e, assim, foi autorizada a substituição de aulas 
presenciais por aulas em meios digitais, pelo Comitê Operativo de Emergência do Ministério da Educação - COE-MEC (BRASIL, 2020). Considerando esses dados, interessa a esse estudo analisar como estudantes universitários expressam ansiedade, diante do Ensino Remoto Emergencial, durante a pandemia da Covid-19, a partir dos tweets publicados nesse período.

\section{METODOLOGIA}

Trata-se de um estudo qualitativo, documental, de natureza exploratória. A pesquisa do tipo documental debruça-se sobre a coleta e exame de documentos que ainda não foram estudados ou tratados anteriormente sobre uma perspectiva de análise e cientificidade. Logo, seu objetivo de compreensão acerca de um fenômeno se configura por meio da extração diretamente da matriz (HELDER, 2006), para que, do contato com a matéria prima, sejam propostas medidas intelectivas e operativas, seja sobre a complementação de informações ou de interpretações.

Ratificando, portanto, a condição primária documental e sua importância na avaliação e captação das vivências, utilizou-se o Twitter, em seu caractere midiático e social, com o objetivo de conhecer como os estudantes universitários experienciam a realidade da implantação do Ensino Remoto na pandemia, viabilizando um contato autêntico com as emoções emanadas e expressas pelos tweets.

Isto posto, foram aplicados como critérios inclusivos para seleção e análise tweets com publicação entre o período de março de 2020 a março de 2021, de usuários com possível identificação de estado e gênero e discentes do âmbito universitário. Como critérios de exclusão, foram utilizados: publicações fora do período estipulado, perfis que sejam privados ou de estudantes que não sejam universitários, endereçados a uma pessoa ou instituição específica e publicações remetentes de federações estrangeiras.

Os tweets foram coletados através da API pública do Twitter de forma automática, utilizando-se scripts de coleta em $\mathrm{R}$, a partir de um conjunto de palavraschave: Ansiedade, Pandemia, EAD. A busca inicial detectou 785 publicações, que após aplicações dos critérios acima e análise de dados, finalizou em 53 postagens, que nos possibilitaram investigações acerca da construção e do conteúdo exposto, fazendo um interlocução entre a literatura específica e o sofrimento dos usuários. 


\section{RESULTADOS E DISCUSSÃO}

\section{Covid-19 e suas implicações ansiogênicas}

Tratando-se de uma síndrome respiratória grave, ocasionada pelo vírus SARSCoV-2, a Covid-19, apresenta seu quadro sintomatológico similar a um perfil gripal, no entanto, devido sua mutação mais recente acarreta um agravamento dos sintomas, podendo levar a óbito (SCHUCHMANN et al, 2020), são eles: febre, distúrbios respiratórios, tosse seca, coriza, alterações gastrointestinais, gustativas, olfatórias, além de dores no corpo, fadiga e indisposição. Com seu primeiro caso em dezembro de 2019 em Wuhan - CN (SIFUENTES-RODRÍGUEZ E PALACIOS-REYES, 2020), logo se alastrou por outros países, decretando, Estado de Pandemia Mundial, em março de 2020, pela Organização Mundial da Saúde - OMS (SCHMIDT et al, 2020).

No Brasil, a adoção de medidas drásticas para enfrentamento da Covid-19 apresentou demora, logo instaurou-se as leis de Emergência de Saúde Pública de Importância Nacional e da Quarentena (JUNIOR et al, 2020), todavia, devido a aplicação superficial de contenção, em pouco tempo o vírus já contaminou grande parte da população. Assim, os índices de mortalidade cresceram rapidamente, havendo um enrijecimento das diligências sociais, o isolamento, o lockdown e distanciamento, evitando assim possíveis aglomerações (OPAS, 2020), e com isso, gerando sentimentos de solidão e exílio.

Com isto, instalou-se um período de grandes incertezas e desentendimentos entre a comunidade científica e as autoridades políticas federais, corroborando para um enredo de desorientação, rapidamente difundido pelas mídias sociais como referências inquestionáveis se tornando um terreno fértil para um adoecimento psicológico, que pode ocasionar graves consequências para a saúde mental dos indivíduos (SACRAMENTO E PAIVA, 2020), instaurando, conquanto, um caos iminente e generalizado na sociedade (JUNIOR ET. AL. 2020).

Logo, como consequência circunstancial da situação pandêmica, atenta-se para uma nociva evolução de quadros ansiosos, na qual antes os que se apresentavam de caráter adaptativo, ou seja, permeado pela funcionalidade e naturalidade do desenvolvimento humano (SADOCK, SADOCK E SUSSMAN, 2017), tornam-se 
desadaptativos, com respostas disfuncionais sintomatológicas com alto nível de persistência e possibilidades de risco (DSM-V, 2014). Substitui-se o sentido de proteção e evitação de ameaças, por vivências danosas de transtornos e adoecimentos.

Corroborando, portanto, com Rosa (2020) ao concluir em sua pesquisa que " $80 \%$ dos brasileiros tornaram-se mais ansiosos na pandemia do novo coronavírus", e também ratificado a partir da análise de conteúdo dos tweets atribuídos a \#ansiedade, na qual 30\% dos usuários apontaram sintomatologias iniciais de Transtornos de Ansiedade.

\section{0 impacto pandêmico no ambiente universitário: ansiedade em foco}

Os acometimentos causados pelo vírus Sars-Cov 19 foram muito além das instituições de saúde, adentrando as outras esferas humanitárias, como as sociais e de ensino, assim o a aplicação do isolamento social interferiu diretamente sobre o processo de aprendizagem de indivíduos inseridos no sistema de ensino superior.

Toda essa adversidade, porém, acentuou vulnerabilidades antes já vividas no ambiente universitário e um grande exemplo é o agravamento de Transtornos Mentais Menores (TMM), em principal o da Ansiedade:

IMAGEM 1. llustração do tweet (usuário 02)

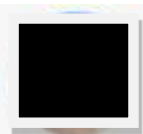

Eu lembrando como era bom quando eu não sofria de ansiedade, nem tinha que trabalhar ou sofrer com EAD

Fonte: Twitter, 2021

IMAGEM 2. llustração do tweet (usuário 03)

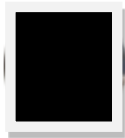

18 de set de 2020

Replying to

eu swi que eu tenho ansiedade (diagnosticada), mas tda eu nunca procurei saber

e parece que só pioraram os "sintomas" depois do ead

Fonte: Twitter, 2021

Costa et. al. (2017), por sua vez, verificaram que não só os fatores anteriores ao período universitário eram agravadores para 0 desenvolvimento destas disfuncionalidades, mas também os vivenciados no próprio ambiente seriam favorecedores para este surgimento. Em vista disso, afirma que o contexto acadêmico é 
desenvolvedor e propagador de "situações desgastantes que podem influenciar ou desencadear o desenvolvimento da ansiedade" (COSTA ET. AL, 2017). As situações se caracterizam por "cargas excessivas de estudo, distanciamento/aproximação do convívio familiar, pressão em trabalhos e provas, além da formação exaustiva de expectativas profissionais" (LELIS ET. AL, 2020). E com o advento da Covid-19, instaurouse mais um agravante.

IMAGEM 3. Ilustração do tweet (usuário 18)

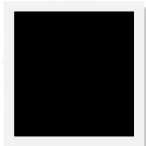

\section{2 de nov de 2020}

o pior dessas aulas em EAD é que elas me obrigaram a ficar em casa e logo conviver mais com ela, resultado disso: emagreci, tive mais crise de ansiedade e autoestima baixa.

Fonte: Twitter, 2021

Dentre as inevitabilidades do período de pandemia a nível educacional, podemos destacar a cessação das atividades das instituições sociais e de ensino na qual afetou diretamente 1,5 bilhão de estudantes de 191 países (UNESCO, 2020). Logo, segundo Pereira, Narduchi e De Miranda (2020), percebeu-se uma necessidade de adoções emergenciais de funcionamento organizacional, desenvolvendo assim, estudos e plano para continuidade da educação (CORDEIRO, 2020), estabelecendo assim o de Ensino Remoto Emergencial (ERE), como única maneira de resolução paliativa da realidade presente (FILHO, ANTUNES E COUTO, 2020).

Em uma pesquisa proposta por Maia e Dias (2020, p.5), em que foram avaliados a presença e os níveis de transtornos ansiosos em acadêmicos, aplicado antes e após o fechamento total das instituições, observou-se "um aumento significativo de perturbação psicológica entre os estudantes universitários no período pandêmico comparativamente a períodos normais." Logo, associou-se à nova forma de ensino a esta causalidade. Perspectiva esta que se reafirmou em tweets, como:

IMAGEM 4. llustração do tweet (usuário 08)

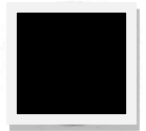

27 de abr de 2020

Gente, eu preciso perguntar se só eu que fui atingido ao ser retirado de um graduação presencial e ser enfiado no ead? pq tipo assim, eu to ouvindo esses professores falando da ansiedade "normal"

Fonte: Twitter, 2021 
Deste modo, soma-se as experiências lesivas de ansiedade da covid-19, com as contingências sociopolíticas e sanitárias advindas da pandemia, afirmadas anteriormente como o fechamento das instituições sociais de ensino, o ERE, renda, acesso aos meios de comunicação e o isolamento/distanciamento social, proporcionando a explosão das expressões emocionais nas mídias sociais.

\section{A exteriorização das vivências ansiosas do ensino remoto e o Twitter}

Advindas com a conjuntura da tecnologia da informação, as mídias sociais, configuram-se de modo a estabelecer uma rede de comunicação informativa seja ela de qual caráter compositivo (SANTOS E MATOS, 2014). Todavia, este modelo interacional, de acordo com as novas necessidades de uso, evolui sua perspectiva comunicativa entre os usuários, permitindo uma nova caracterização da conexão indivíduo-sistema na qual tornou-se um caractere mediar entre pessoa-grupo social (SANTAELLA E LEMOS, 2010), ou seja, a era da Web 2.0 (RUFINO, TABOSA E NUNES (2010)

Assim, adquirindo uma categoria dialógica, a rede secundariza a transmissão de conhecimentos, tornando primária a comunicação multi-sensitiva e monóloga do sujeito (MANESS, 2007), sobre suas vivências pessoais, logo, são exemplos: blogs, podcasting e as diversas redes sociais (ROESLER, 2019). Proporcionando, um alto crescimento destas plataformas, caminhando assim para um expressionismo de rotinas diárias, sentimentos e subjetividades, sendo elas: Facebook, Instagram, Twitter, dentre outras. (SHIMAZAKI E PINTO, 2016).

Sendo, portanto, a base de pesquisa do estudo, a rede do Twitter, iniciada no ano de 2006, caracteriza-se como um microblog que tem como principal objetivo a sintetização de experiências latentes, na qual limita o tweet a 140 caracteres, proporcionando "intercâmbio de ideias e informações entre seus usuários" (TWITTER, 2017), que se dispõem por meio de hashtags (\#) quando voltado a uma pauta específica ou arroba (@) quando endereçada a um outro perfil. Assim, política de caracteres limitados e a seleção de termos chaves tanto para pesquisa, quanto para o tweet público, propicia compreensões diversificadas e de maior intensidade para os receptores. 
Silva (2018), em sua pesquisa acerca da manifestação do Ethos (aspecto intrínseco da pessoalidade) no Twitter, afirmou que, por vezes, a exposição deste componente emotivo do presente, associa-se a tentativa de emissão desta individualidade que se encontra deslocada de seu sentido motivador natural, agora atrelada a uma melancolia. Logo, essa anunciação (deste Ethé) discorre sobre uma tentativa do enunciador de resolução, ajuda ou propagação. Contudo, devido as condições ambientais de nível social, acaba transpondo sobre um processo de adesão tornando o outro um coenunciador. Fatos estes que podem ser avaliados por meios dos discursos conectados pelo mecanismo de "retweet" (republicação), "replying to" (respostas) ou até likes em threads:

IMAGEM 5. Ilustração do tweet (usuário 20)

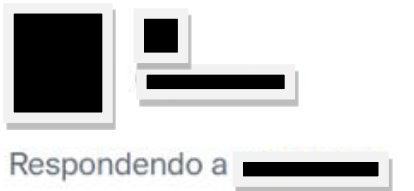

\section{4 - teve alguma coisa que você aprendeu no ead?} Traduzir Tweet

$12 \mathrm{~h} 43 \cdot 20$ de fev de 2021 . Twitter para iPhone

\section{Retweets $12,5 \mathrm{~K}$ Citar Tweets 963 Gosta}

Fonte: Twitter, 2021

Por consequência, este expressionismo, ultrapassa as barreiras de remetentedestinatário, atingindo sujeitos não diretamente indexados e que também se apropriam daquela perspectiva. Assim, estas manifestações tornam o "agora" não só de um sujeito, mas de uma rede (MANDEL ET. AL, 2012). Logo, este compartilhamento massivo, adquire um padrão desadaptativo de uso, proporcionando a toda a rede sofrimentos compartilhados e adoecimentos psíquicos em massa (CASANI ET AL, 2020). Como afirma também Bueno (2018), ao comparar a utilização disfuncional das redes sociais com o surgimento de comportamentos ansiosos e depressivos, e de negligência da vida.

IMAGEM 6. Ilustração do tweet (usuário 22)

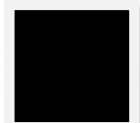

- 11 de fev

Uma curtida e eu desisto do Ead e da vida.

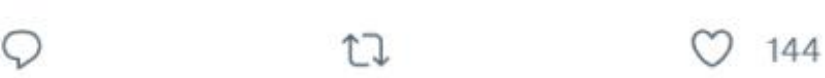




\section{CONSIDERAÇÕES FINAIS/CONCLUSÕES}

Os impactos causados pela Covid-19, portanto, transpassam as esferas biológicas e adentram de forma disfuncionais nos aspectos biopsicossociais, que envolvem não só um sujeito, mas toda uma massa. Com isso, os altos índices de ansiedade, tornam-se ainda mais alarmantes quando observados sobre uma perspectiva a longo prazo, haja vista que seus danos evadem de uma condição específica do sujeito, adentrando sobre suas áreas de desenvolvimento e expansão.

O ambiente acadêmico, antes, visto e experienciado sob uma óptica de criação e construção, torna-se eliciador de vivências ansiogênicas e adoecedoras. Logo, suas repercurssões alertam grande necessidade de intervenção afim de proporcionar ressignificação de estímulos e comportamentos, afim de que diminua os riscos de danosas implicações no eu profissional, em formação.

\section{REFERÊNCIAS}

BUENO, Ana Cláudia et al. Uso da rede social FacebookTM na coleta de dados e disseminação de evidências. Escola Anna Nery, v. 22, n. 3, 2018. Disponível em: < https://www.scielo.br/scielo.php?pid=S141481452018000300209\&script=sci_arttext\& tlng=pt > Acesso em: 21 fev 2021.

CASANI, Vinicius et al. Identificação de Perfis Depressivos em Redes Sociais Utilizando Aprendizado de Máquina: um Mapeamento Sistemático. Anais do Computer on the Beach, v. 11, n. 1, p. 183-190, 2020. Disponível em:< https://www.researchgate.net/profile/Francisco-Carlos-Souza2/publication/344158962_Identifying_Depressive_Profiles_on_Social_Networks_using _Machine_Learning_A_Systematic_Mapping/links/5f56c06692851c250b9cee8f/Identif ying-Depressive-Profiles-on-Social-Networks-using-Machine-Learning-A-SystematicMapping.pdf> Acesso em: 21 mar. 2021.

CASTILLO, Ana Regina GL et al. Transtornos de ansiedade. Brazilian Journal of Psychiatry, v. 22, p. 20-23, 2000. Disponível em: https://www.scielo.br/scielo.php?pid=S1516-44462000000600006\&script=sci_arttext. Acesso em: 22 fev. 2021

CORDEIRO, Karolina Maria de Araújo. O Impacto da Pandemia na Educação: A Utilização da Tecnologia como Ferramenta de Ensino. 2020. Disponível em: http://repositorio.idaam.edu.br/jspui/handle/prefix/1157. Acesso em: 25 fev. 2021. 
COSTA, Kercia Mirely Vieira et al. Ansiedade em universitários na área da saúde. In: II Congresso Brasileiro das Ciências da saúde. 2017. Disponível: < https://www.editorarealize.com.br/editora/anais/conbracis/2017/TRABALHO_EV071_ MD1_SA13_ID592_14052017235618.pdf>. Acesso em: 25 fev. 2021.

DA SILVA, Albylene. Ethos de melancolia em postagens pessoais no Twitter. MiguilimRevista Eletrônica do Netlli, v. 6, n. 3, p. 113-137, 2018.

FILHO, Astrogildo Luiz; ANTUNES, Charlles; COUTO, Marcos Antonio Campos. Alguns apontamentos para uma crítica da EAD na educaçã:o brasileira em tempos de pandemia. Revista Tamoios, v. 16, n. 1, 2020. Disponível em: < https://www.epublicacoes.uerj.br/index.php/tamoios/article/view/50535>. Acesso em: 26 fev. 2021.

HELDER, R. R. Como fazer análise documental. Porto, Universidade de Algarve, v. 1, p. 1-5, 2006.

JÚNIOR, João Henrique de Sousa et al. Da Desinformação ao Caos: uma análise das Fake News frente à pandemia do Coronavírus (COVID-19) no Brasil. Salvador, BA: Cadernos de Prospecção, 2020. 331-346 p. Disponivel em: < https://periodicos.ufba.br/index.php/nit/article/view/35978>. Acesso em: 23 fev. 2021.

LELIS, Karen de Cássia Gomes et al. Sintomas de depressão, ansiedade e uso de medicamentos em universitários. Rev. port. enferm. saúde mental, p. 9-14, 2020. Disponível em: <

https://web.a.ebscohost.com/abstract?direct=true\&profile=ehost\&scope=site\&authty pe $=$ crawler\&jrnl $=16472160 \& A N=144426178 \& h=F v q m J X n V 5 x N m Z j 4 L h j l j i b A O a T X c \% 2 f f$ EKjr9FKZOozRxWliLkHXWtOXAdt9yF5IpqI\%2fFINYy4HtBNidPFriZWEw\%3d\%3d\&crl=c\& $r$ esultNs=AdminWebAuth\&resultLocal=ErrCrINotAuth\&crlhashurl=login.aspx\%3fdirect\% 3dtrue\%26profile\%3dehost\%26scope\%3dsite\%26authtype\%3dcrawler\%26jrnl\%3d164 72160\%26AN\%3d144426178>. Acesso em: 25 fev. 2021.

MAIA, Berta Rodrigues; DIAS, Paulo César. Ansiedade, depressão e estresse em estudantes universitários: o impacto da COVID-19. Estudos de Psicologia (Campinas), v. 37, 2020. Disponível em:< https://www.scielo.br/scielo.php?script=sci_arttext\&pid=S0103166X2020000100504\&tIng=pt.> Acesso em: 26 fev. 2021.

MANDEL, Benjamin et al. A demographic analysis of online sentiment during hurricane irene. In: Proceedings of the second workshop on language in social media. 2012. p. 27-36. Disponível em: < https://www.aclweb.org/anthology/W12-2104.pdf>. Acesso em: 21 fev. 2021.

MANESS, Jack M. Teoria da biblioteca 2.0: web 2.0 e suas implicações para as bibliotecas. Informação \& Sociedade, v. 17, n. 1, 2007. Disponível em: < https://brapci.inf.br/_repositorio/2010/11/pdf_d1b75c96ad_0012775.pdf>. Acesso em: $11 \mathrm{fev} / 2021$.

Organização Pan-Americana de Saúde (OPAS-Brasil). (2020). Folha informativa COVID-19 (doença causada pelo novo coronavírus). Disponível em: < 
https://www.paho.org/pt/covid19\#: :text=É\%20preciso\%20seguir\%20e\%20adotar,lixo \%20e\%20lave\%20as\%20mãos).> . Acesso em: 23 fev. 2021.

PEREIRA, Alexandre de Jesus; NARDUCHI, Fábio; MIRANDA, Maria Geralda de. BIOPOLÍTICA E EDUCAÇÃO: os impactos da pandemia do covid-19 nas escolas públicas. Revista Augustus, [S.L.], v. 25, n. 51, p. 219-236, 3 jun. 2020. Sociedade Unificada de Ensino Augusto Motta -UNISUAM. http://dx.doi.org/10.15202/1981896.2020v25n51p219. Disponível em: https://sagaweb.unisuam.edu.br/index.php/revistaaugustus/article/view/554. Acesso em: 25 fev. 2021.

ROESLER, Rafael. Web 2.0, interações sociais e construção do conhecimento. VII SIMPED-Simpósio Pedagógico e Pesquisas em Educação-2012. v. 10, n. 09, 2019. Disponível em: <https://www. aedb. br/wp-content/uploads/2015/04/45817495.pdf> Acesso em: 11 fev. 2020

ROLIM, Josiane Alves; DE OLIVEIRA, Aldecir Ramos; BATISTA, Eraldo Carlos. Manejo da ansiedade no enfrentamento da Covid-19. Revista Enfermagem e Saúde ColetivaREVESC, v. 5, n. 1, p. 64-74, 2020. Disponível em:

https://www.researchgate.net/profile/Josiane-

Rolim/publication/343678426_Manejo_da_Ansiedade_no_Enfrentamento_da_Covid19_Managing_Anxiety_in_Coping_with_Covid19/links/5f3827be299bf13404c8490a/Manejo-da-Ansiedade-no-Enfrentamento-daCovid-19-Managing-Anxiety-in-Coping-with-Covid-19.pdf. Acesso em: 25 fev. 2021

RUFINO, Airtiane Francisca; TABOSA, Hamilton Rodrigues; NUNES, Jefferson Veras. Redes sociais: surgimento e desenvolvimento dos micro-bloggings. 2010. Disponível em: < http://repositorio.ufc.br/handle/riufc/22674> . Acesso em: 11 fev. 2021

SACRAMENTO, I.; PAIVA, R. Fake news, Whatsapp e a vacinação contra febre amarela no Brasil. São Paulo, v.14, n.1 p.79-106, jan./abr. 2020. Disponível em: < https://www.revistas.usp.br/matrizes/article/view/160081>. Acesso em: 23 fev. 2021.

SADOCK, Benjamin; SADOCK, Virginia A.; SUSSMAN, Norman. Kaplan \& Sadock's pocket handbook of psychiatric drug treatment. Lippincott Williams \& Wilkins, 2017.

SANTAELLA, Lúcia; LEMOS, Renata. Redes sociais digitais: a cognição conectiva do Twitter. São Paulo: Paulus, v. 137, 2010. Disponível em: < https://revistas.uca.es/index.php/hachetetepe/article/download/6393/6496/26219>. Acesso em: 11 fev. 2021.

SCHMIDT, B et al. Impactos na saúde mental e intervenções psicológicas diante da pandemia do novo coronavírus (covid-19). Disponível em:< https://doi.org/10.1590/SCIELOPREPRINTS.58> Acesso em:23 fev 2021.

SCHUCHMANN, Alexandra Zanella et al. Isolamento social vertical $\mathbf{x}$ isolamento social horizontal: os dilemas sanitários e sociais no enfrentamento da pandemia de COVID19. Disponível em: <\&lt; https://doi.org/10.34119/bjhrv3n2-185 \&gt;> Acesso em: 16 fev 2021. 
SHIMAZAKI, Vinicius Kenji; PINTO, Maria Márcia Matos. A influência das redes sociais na rotina dos seres humanos. FaSCi-Tech, v. 1, n. 5, 2016. Disponível em:

http://fatecsaocaetano.edu.br/fascitech/index.php/fascitech/article/view/57. Acesso em: 11 fev. 2020.

SIFUENTES-RODRÍGUEZ, Erika; PALACIOS-REYES, Deborah. COVID-19: The outbreak caused by a new coronavirus. Boletin Medico del Hospital Infantil de Mexico, v. 77, n. 2, p. 47-53, 2020. Disponível em:

<http://www.scielo.org.mx/scielo.php?script=sci_arttext\&pid=S166511462020000200 047>. Acesso em: 23 fev. 2021.

TWITTER. Company. 2017. Disponível em: https://about.twitter.com/company. Acesso em 21 fev. 2021.

UNESCO. United Nations Educational, Scientific and Cultural Organization. Global Monitoring of school closures caused by COVID-19. Disponível em:

https://en.unesco.org/covid19/educationresponse. Acesso em: 25 fev. 2021.

Zarocostas, J. How to fight an infodemic. The Lancet, 395(10225), 676, 2020. 